Fortbildungsveranstaltung

\title{
Misteltherapie in der
}

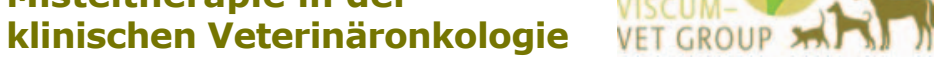

Inhalte

- Praktische Anwendung bei Hund, Katze und Pferd

- Aktuelle Studienlage

- Grundlagen, Immunologie und Therapieprotokolle

Kursdetails

Mi: 08. November 2017 14:00-17:30 Uhr

Do: 09. November 2017 09:00 - 17:00 Uhr

Am FiBL in Frick (Schweiz)

Weitere Informationen und Anmeldung unter www.fibl.org

\section{KARGER}

\section{Hier könnte Ihr Fort- und Weiterbildungsangebot stehen!}

Nutzen Sie SCHWEIZERISCHE ZEITSCHRIFT FÜR GANZHEITSMEDIZIN zur effektiven Kommunikation mit Ihrer Zielgruppe! So schaffen Sie die besten Voraussetzungen sowohl gedruckt als auch online gesucht und gefunden zu werden.

Informationen und Angebote bei: Petra Schlegel

Tel. +49 761 45207-13

p.schlegel@karger.com

"Ihr Aus- und Weiterbildungsinstitut IKP:
wissenschaftlich - praxisbezogen - anerkannt "
Dr. med. Yvonne Maurer
Anerkannte psychotherapeutische Jahren anerkannt
Weiterbildung im Rahmen Fachärztin/Facharzt
FMH für Psychiatrie und Psychotherapie
Körperzentrierte Psychotherapie IKP
Die Körperzentrierte Psychotherapie IKP nach Dr. Dr. Yvonne Maurer vereint Elemente
aus der Gestalt- und Körperpsychotherapie sowie systemische Ansätze. Die
ganzheitliche Methode berücksichtigt alle zentralen Lebensdimensionen.

